\title{
Penser et vivre la frontière dans les relations franco-allemandes
}

Grenzen bestimmten lange Zeit die deutsch-französischen Beqiehungen und haben folgenschwere Konflikte zwischen den beiden Nachbarvölkern ausgelöst. In deren Mittelpunk.t stand naturgemäß der Rhein, der einen Teil der deutsch-französischen Grenze ausmacht und über die Jahrbunderte eine emotionale und nationale Aufladung erbielt, welche starke Antagonismen zutage gefördert hat. Nachdem sich die Spannungen im Laufe des 19. Jabrhunderts verstärkt hatten und in einem ersten kriegerischen Konflikt sowie den katastrophalen Ereignissen des 20. Jahrbunderts gipfelten, war ein anderes Grenzdenken vonnöten, um den Völkern ein neues Miteinander zu ermöglichen. Aber wie konnte aus dieser, historischen Narbe' ein dauerhaft stabiles Bündnis zwischen den beiden Ländern im Herzen Europas entstehen?

Der vorliegende Beitrag setzt beim veränderten Grenzempfinden im Rahmen der deutsch-französischen Beziehungen nach 1945 an. Äbnlich wie das Bild von der, Erbfeindschaft" musste auch die deutsch-französische Grenze politisch, militärisch, kulturell und mental demobilisiert werden, um den ,Schnitt', den der Rhein dargestellt hatte, in eine ,Nabt' umzuwandeln und diesen ,Nil des Okzidents' von einer Grenzlinie zu einem europäischen Beziehungsraum werden zu lassen. Inwiefern war die Grenznähe dabei ein $V$ orteil oder aber, zumindest in der ersten Zeit, ein Hindernis für die bilateralen Bestrebungen einer Annäherung?

Ausgebend von einer historischen Auseinandersetzung mit dem Grenzbegriff unter Gegenüberstellung der Konzeptionen einer, natürlichen Grenze; eines deutschen, französischen oder europäischen Rheins, zeigt dieser Beitrag, wie es Franzosen und Deutschen gelungen ist, mit dieser schmerzhaften Vergangenheit abzuschließen, indem sie nach 1945 den grenzüberschreitenden Kontakt und Dialog gesucht haben. Folglich werden die beiden Länder heute oft als Vorzeigemodell für eine gelungene Versöhnung verstanden und die grenzüberschreitende bilaterale Kooperation ist zum Aushängeschild sowie zu einem spannenden Erprobungsfeld ihrer Beziehung geworden.

«La cicatrice de l'histoire/die Narbe der Geschichte ». C'est ainsi qu'en 1973 Alfred Mozer, journaliste et homme politique germano-néerlandais, qualifia la frontière franco-allemande qu'il voulait transformer en un foyer de coopération locale et régionale. ${ }^{1}$ Aujourd'hui, 50000 travailleurs frontaliers se rendent

1 Mozer, Alfred : Entwicklungspolitik zu Hause, ds. : Schöndube, Claus (dir.) : Entwicklungsregionen in der EWG, Bonn: Bundeszentrale für politische Bildung (Schriften der Bundeszentrale für politische Bildung), 1973, p. 14-25, ici p. 14, cité d'après Wassenberg, Birte : 
de France en Allemagne (dont un quart de résidents allemands en France) et 4000 Allemands viennent chaque jour en France. ${ }^{2}$ Les $451 \mathrm{~km}$ de frontière passant entre trois Länder allemands et trois départements français sont un lieu d'échanges permanents et intenses. Les ponts sur le Rhin, à Strasbourg notamment, canalisent la circulation des flux et sont aussi un symbole du lien : «pont de l'Europe », inauguré en 1960 en symbole du rapprochement bilatéral; " passerelle des deux rives » créée en 2004 ; nouveau pont de chemin de fer ouvert en 2010 et, pont du tram reliant Kehl et Strasbourg, depuis le printemps 2017. Le pont entre la France et l'Allemagne est devenu l'un des emblèmes de l'histoire bilatérale. En 2003, pour le 40e anniversaire du traité de coopération franco-allemande, Tomi Ungerer a dessiné un timbre représentant un pont reposant sur des pieds 'humains', dépassant le fossé rhénan, double évocation de la frontière et du fossé de l'histoire. Plus que le rapprochement, il dépeint une histoire d'amour sur toile de fond européenne. C'est un condensé iconographique du grand récit de la réconciliation franco-allemande! Près d'un siècle plus tôt, la France et l'Allemagne étaient en guerre et la question des frontières avait été l'un des buts de guerre de la France. Le pont a aussi été souvent un symbole de dissociation (l'expulsion de 100000 Allemands en 1918/19), de séparation (le pont fermé au lendemain de la Grande Guerre) et de la guerre elle-même (le pont de Strasbourg, détruit en 1940).

L'histoire des relations franco-allemandes est donc pour partie une histoire des relations à la frontière. Nous nous proposons de saisir le retournement de la valeur de la frontière dans ces relations après 1945 - symboliquement, on pourrait dire du Rhin - même s'il ne s'agit évidemment pas de cette seule frontière. Dans quelle mesure le facteur de proximité ou d'éloignement par rapport à la frontière a-t-il été un atout pour l'engagement bilatéral ? Comment la frontière a-t-elle été et reste-t-elle perçue par ceux qui y vivent? Avant d'examiner la phase du rapprochement franco-allemand et celle de la coopération entre des voisins 'réconciliés' et 'amis' - au cours de laquelle les régions frontalières sont devenues une vitrine - il faut jeter un regard sur un passé plus lointain, où se sont succédé différentes conceptions de la frontière qui ont fini par cristalliser l'antagonisme franco-allemand et ont affecté profondément le quotidien des populations locales.

Grenzorte. Von der Konfrontation zur Kooperation. Das Beispiel des Oberrheins, ds. : Defrance, Corine/Pfeil, Ulrich (dir.) : Verständigung und Versöbnung nach dem „Zivilisationsbruch"? Deutschland in Europa nach 1945, Bruxelles : Peter Lang, 2016, p. 349-368.

2 Mission Opérationnelle Transfrontalière: Frontière France-Allemagne, http://www. espaces-transfrontaliers.org/ressources/territoires/frontieres/frontieres-en-europe/ frontiere-france-allemagne/frontiere-france-allemagne-2/ (10/01/2017). 
1. Frontière et idée de la frontière jusqu'à la Deuxième Guerre mondiale

\subsection{L'établissement de la frontière franco-allemande : le cas emblématique du Rhin}

Comme l'a souligné l'historien Daniel Nordman, il n'y a pas de frontières naturelles, mais seulement une théorie des frontières naturelles. ${ }^{3}$ L'idée de 'frontière naturelle' est une construction relativement tardive, qui a pour but d'ancrer dans la géographie la coupure politique. C'est une manière de légitimer, par le recours à l'évidence de la nature, les identités nationales.

Au XVIe siècle et pendant une bonne partie du XVIIe siècle encore, la revendication d'une frontière sur le Rhin avait été rare. ${ }^{4}$ Richelieu ne plaidait pas pour une frontière rhénane linéaire, mais entendait s'assurer des têtes de pont sur le Rhin et des voies d'accès vers l'intérieur de l'Empire. Progressivement, au cours du XVII siècle, émergea la notion de 'limite naturelle'. L'idée a notamment circulé dans les manuels d'histoire et de géographie rédigés par les jésuites, véhiculant l'image d'une France bornée par la mer, les Pyrénées et le Rhin en référence à la Gaule de César. Avec les traités de Westphalie (1648), une grande partie de l'Alsace passa sous souveraineté française, mais les limites en restèrent floues et contestées, incluant des territoires sur la rive droite du Rhin. La situation évolua avec l'annexion de Strasbourg en 1681 puis surtout avec la paix de Ryswick entre la France et le Saint-Empire (1697) : «A Ryswick, la construction de la France est parvenue à son terme sur la ligne du Rhin $»^{5}$. Mais c'est surtout à partir de 1793 et la proclamation de Danton selon laquelle les limites de la France seraient marquées par la nature que s'est ancrée l'idée de 'frontières naturelles' qui devait connaître une popularité considérable au siècle suivant. En 1798, quatre départements français furent créés sur la rive occidentale du Rhin. Mais en 1806, suite à la formation de la Confédération du Rhin, étroitement liée à l'Empire napoléonien, le Rhin perdit sa fonction de frontière et devint quasiment un fleuve français. En 1815, au congrès de Vienne, la frontière de 1792 fut rétablie. Le Rhin redevient la 'frontière naturelle' au niveau de l'Alsace et du Grand-Duché de Bade, puis un 'fleuve allemand' en aval, mais de part et d'autre les revendications ne s'apaisèrent pas : Rhin allemand ('deutscher Strom') contre 'frontière naturelle' de la France. Avec la Prusse désormais installée sur les rives du fleuve et l'essor industriel et économique des régions rhénanes, l'instrumentalisation

3 Nordman, Daniel : Frontières de France. De l'espace au territoire. XV Te-XIXe siècles, Paris : Gallimard, 1998, p. 11.

4 Zeller, Gaston : Aspects de la politique française sous l'Ancien Régime, Paris : PUF, 1964.

5 Nordman : Frontières de France, p. 311. 
politique du Rhin s'amplifia. ${ }^{6}$ D'un point de vue géopolitique, la situation ne changea qu'au lendemain de la guerre de 1870/71. Le Rhin devint un fleuve allemand. En France, on se mobilisa pour recouvrer les 'provinces perdues' (Alsace et une partie de la Lorraine). Avec le traité de Versailles de 1919, on en revint au Rhin frontière sur son cours supérieur et au Rhin allemand au nord de l'Alsace, mais avec l'hypothèque de l'occupation qui amena la France et ses alliés sur le Rhin, voire au-delà du Rhin (cf. l'occupation de la Ruhr notamment). En 1930, l'occupation de la rive gauche du Rhin prit fin, mais le Rhin resta en zone démilitarisée. L'entrée de la Wehrmacht sur la rive gauche du Rhin en mars 1936 fut un triomphe pour la revendication du 'Rhin allemand'.

La Seconde Guerre mondiale reposa immédiatement la question des frontières. Reprit le cycle de l'annexion de facto (Alsace/Moselle en 1940), puis, après la guerre, de l'occupation - notamment par la France -, cette fois-ci sur les deux rives du Rhin, et des incertitudes sur l'avenir de la Sarre jusqu'au référendum de 1955. Depuis le retour de la Sarre à la République fédérale, la frontière franco-allemande est une frontière fixée et acceptée, qui lie bien plus qu'elle ne sépare. De coupure, elle est devenue couture. Avant d'en venir à la frontière passerelle, arrêtons-nous sur la frontière comme moteur et lieu de cristallisation de l'antagonisme franco-allemand.

\subsection{Le Rhin, symbole de l'antagonisme franco-allemand: construction et diffusion d'un grand récit}

Au début du XIXe siècle, en plein romantisme, le Rhin était loué par les peintres et poètes, allemands et étrangers. Mais bientôt, en réaction aux conquêtes napoléoniennes et aux revendications du Rhin 'frontière naturelle' de la France voire du 'Rhin français', les combattants allemands des 'guerres de libération' se mobilisèrent pour défendre le 'Rhin allemand' à l'instar de Ernst Moritz Arndt selon l'argument que la seule frontière naturelle serait celle de la langue. ${ }^{7}$ Après avoir un temps été fasciné par la France révolutionnaire, il fut l'un de ceux qui attisa le sentiment antifrançais.

En 1840, la résurgence de revendications françaises sur la rive gauche du Rhin provoqua un nouveau choc des nationalismes qu'illustre la 'guerre des poètes'. Nicolaus Becker, dans Der freie Rhein (1840), s’écria : «Ils ne l'auront

6 Cf. Suckow, Dirk : Deutscher Rhein, französischer Rhein, ds. : Bundeszentrale für politische Bildung: Geschichte im Fluss, Flüsse als europäische Erinnerungsorte, 11/05/2012, http:/ / www.bpb.de/geschichte/zeitgeschichte/geschichte-im-fluss/135684/deutscher-rheinfranzoesischer-rhein?p=all3 (10/01/2017).

7 Suckow : Deutscher Rhein, französischer Rhein. 
pas le libre Rhin allemand $»^{8}$, à quoi Alfred de Musset répliqua « Nous l'avons eu, votre Rhin allemand. Il a tenu dans notre verre $»^{9}$, dans un bref poème d'une rare brutalité (Le Rhin allemand, 1841). La réponse de Lamartine - La Marseillaise de la Paix -, à l'époque jugée trop molle par ses concitoyens, mit en lumière le Rhin comme fleuve de la paix liant les peuples. Il rêvait du Rhin qui réconcilie, irriguant une civilisation et la culture européenne. Le Rhin devint sous sa plume le «Nil de l'Occident $»^{10}$. Ce fut aussi la vision de Victor Hugo dans Le Rhin, lettres à un ami, paru en 1842. Ces poètes français répliquèrent donc par l'image d'un Rhin européen et pacifique - ce qui était une idée très française. Dans le sillage de cette 'crise du Rhin', le fleuve devint un paysage identitaire où confluèrent nature, histoire et culture. ${ }^{11}$ L'étranger proche devint 'l'ennemi national', indispensable à la construction et à l'affirmation de sa propre identité. ${ }^{12}$ Cette 'guerre lyrique' mit en lumière la force de l'émotion en ce milieu de siècle. C'est surtout la postérité de ces poèmes et chants qui est étonnante. Ainsi la Wacht am Rhein de Max Schneckenburger devint l'hymne des armées allemandes pendant la guerre de 1870 et servit encore de modèle pour une 'Garde sur la Somme' des soldats allemands en $1916 .{ }^{13}$

Outre les écrivains et les poètes, les peintres et les sculpteurs mobilisèrent leur art au service de la 'guerre des images'. En 1860, Lorenz Clasen peignit pour la mairie de Krefeld Germania auf der Wacht am Rhein. La seule présence de Napoléon III à Paris rappelait dans une Allemagne pas encore unifiée les souvenirs de Napoléon Ir et des Français sur le Rhin. C'est cette même allégorie de la nation allemande que l'on retrouve au bord du Rhin, près de Rüdesheim, au monument du Niederwald érigé en 1883 pour célébrer la victoire de 1871 contre la France et l'unité allemande. En 1889, les artistes lorrains Émile Gallé et Victor Prouvé répliquèrent par la table monumentale

8 Vgl. Becker, Nicolaus: Der freie Rhein, d'abord ds.: Trierische Zeitung 257, 18/09/1840. Voir aussi Becker, Nicolaus: Gedichte, Köln : DuMont-Schauberg, 1841, p. 216-218.

9 Musset, Alfred de : Le Rhin allemand, in : La Revue de Paris, 06/06/1841.

10 «Roule libre et superbe entre tes larges rives, / Rhin, Nil de l'Occident, coupe des nations! », Lamartine, Alphonse de : La Marseillaise de la Paix, ds. : La Revue des deux Mondes 4/26 (1841), p. 794-799.

11 Walter, François : Les figures paysagères de la nation. Territoire et paysage en Europe (16e-20e siècle), Paris : Editions Ecole des Hautes Etudes en Sciences sociales, 2004.

12 Jeismann, Michael : Das Vaterland der Feinde. Studien zum nationalen Feindbegriff und Selbstverständnis in Deutschland und Frankreich 1791-1918, Stuttgart : Klett-Cotta, 1992 (paru en français sous le titre : La patrie de l'ennemi. La notion d'ennemi national et la représentation de la nation en Allemagne et en France de 1792 à 1918, Paris : CNRS Editions, 1997).

13 Beaupré, Nicolas : Le Rhin. Une géographie, Paris : La documentation française, 2005 (Documentation photographique, dossier $\left.n^{\circ} 8044\right)$, p. 12. 
nommée Le Rhin où l'on voit la figure paternelle du Rhin protéger des Germains une allégorie féminine de la Moselle. Ils revendiquent ainsi l'idée du Rhin 'frontière naturelle' de la France et réclament, en profitant de la visibilité que leur donne l'Exposition universelle, le retour des provinces perdues à la France. Prouvé s'exclame : c'est « la plus belle gifle qu'on puisse leur flanquer pour le moment $»{ }^{14}$

Les historiens usèrent eux aussi de leur science comme d'une arme. Après la Première Guerre mondiale, les historiens allemands participèrent aux diverses campagnes pour une révision du traité de Versailles. Dès 1919 furent créés plusieurs instituts de recherche et d'études le long des frontières du Reich, à Francfort et Bonn notamment, ayant un objectif commun : "Faire le point sur la guerre, sur les nouvelles frontières, les référendums, les pertes de territoire et de population, et donner à tout cela une dimension historique qui remonte le plus loin possible - le Moyen Âge, l'Antiquité -, afin de prouver par la science de l'histoire que les revendications allemandes sont justifiées $\gg^{15}$. Pendant l'entre-deux-guerres, ils pratiquèrent une sorte d'auto-mobilisation autour de la thèse de l'inimitié héréditaire entre la France et l'Allemagne. C'est ce que reflète l'attitude ouvertement francophobe de l'historien Hermann Oncken, déclarant en 1924 : «L'ennemi n'est ni à droite ni à gauche, l'ennemi est sur le Rhin !».16

\subsection{Vivre à la frontière - percevoir la frontière}

A l'époque moderne, alors que la frontière du Rhin était une voie de délimitation, les populations riveraines, relevant de souverainetés politiques différentes, n'étaient pas séparées. La circulation sur le fleuve liait les peuples et toutes sortes de métiers caractéristiques du Rhin donnaient à la région une identité.

14 Beaupré : Le Rhin. Une géographie, p. 28. Voir aussi Perrin, Jérôme : La collaboration entre Emile Gallé et Victor Prouve, ds. : Annales de l'Estn n spécial (2005), p. 199-210 ; Plessen, Marie-Louise von [et al.] : Marianne et Germania. 1789-1889. Un siècle de passions franco-allemandes, Paris : Musée du Petit Palais, 1997.

15 Schöttler, Peter : La Zeitgeschichte allemande, ds. : Écrire l'bistoire 11 (2013), p. 61-68, ici p. 61 ; voir aussi : Schöttler, Peter : Geschichtsschreibung als Legitimationswissenschaft 1918-1945, Frankfurt/M. : Suhrkamp, 1997 ; Freund, Wolfgang: Das wissenschaftliche Institut der Elsass-Lothringer im Reich an der Universität Frankfurt a. M. (1920-1945), ds. : Pfeil, Ulrich (dir.) : Deutsch-französische Kultur- und Wissenschaftsbeziebungen im 20. Jahrbundert. Ein institutionengeschichtlicher Ansatz, München : Oldenbourg, 2007, p. 47-71.

16 Oncken, Hermann : Nation und Geschichte, Berlin : Grote, 1935, p. 197. Voir aussi Pfeil, Ulrich : Eugen Ewig. „Créer un ordre transnational“. Von einem Mittler zwischen Deutschland und Frankreich, ds. : Pfeil, Ulrich (dir.) : Das Deutsche Historische Institut Paris und seine Gründungsväter, München : Oldenbourg, 2007, p. 294-322. 
Des traits culturels et religieux, des modes de vie aussi, constituaient l'espace rhénan. ${ }^{17}$

Même avec l'établissement de la frontière politique sur le Rhin, pendant longtemps, le fleuve n'a pas été perçu par les contemporains comme la séparation entre la France et les États allemands. Dans les récits de voyage de la fin du XVIII e siècle, le passage de la frontière n'est parfois même pas mentionné, et surtout les voyageurs décrivent une absence de rupture : la langue, l'aspect des villages, la façon de s'habiller, les habitudes alimentaires ne se modifiaient que très graduellement. Certains disent que c'est en Lorraine, voire en Champagne seulement, qu'ils ont eu le sentiment d'arriver en France... Ils brossaient ainsi le portrait d'une zone frontière de culture mêlée francogermanique. La perception de la frontière changea radicalement après 1815 : le voyageur éprouvait désormais le passage d'une ligne frontière séparant sa patrie de l'étranger. ${ }^{18}$ Ce n'est pas tant la frontière qui avait changé que sa perception. Pour le voyageur allemand, elle était désormais perçue et vécue à l'aune des guerres de la Révolution puis de l'Empire. Les défaites face aux armées napoléoniennes puis les guerres de libération transformèrent radicalement la perception du voisin et, par voie de conséquence, de la frontière.

Bien sûr, même avant les guerres de la Révolution et de l'Empire, les confins avaient été régulièrement des terres d'invasion, de pillages ou de massacres. Mais l'émergence des identités nationales suscita la prise de conscience d'une domination étrangère dont les violences étaient particulièrement intolérables. Les populations frontalières connurent souvent des destins particulièrement rudes, marqués par les occupations et les annexions. Elles furent souvent suspectées - parce qu'elles étaient géographiquement à la marge et culturellement au contact de l'étranger - de manquer de loyauté et même d'être des traîtres potentiels. Elles furent soumises à des expériences et des violences extrêmes en période de conflit : évacuations, répression, incorporations de force dans l'armée, expulsions. C'est dans ces territoires et en raison $\mathrm{du}$ sort de ces territoires - surtout au cours du premier $\mathrm{XX}^{\mathrm{e}}$ siècle - que grandit la haine de l'autre. La campagne antifrançaise atteignit son acmé lors de l'occupation de la rive gauche du Rhin et de la Ruhr au lendemain de la Première Guerre mondiale. Quelques années plus tard, Hitler remobilisa cette haine antifrançaise. Inversement, les régions frontalières - en raison de leurs expériences spécifiques, ne jouèrent qu'un rôle mineur dans le bref rapproche-

17 Cf. Nordman : Frontières de France, p. 310-311.

18 Cf. Struck, Bernhard/Gantet, Claire : Revolution, Krieg und Verflechtung: 1789-1815, Darmstadt: WBG, 2008, p. 21 (paru en français sous le titre : Révolution, guerre et interférences, 17891815, Villeneuve d'Ascq : PU du Septentrion, 2013). 
ment franco-allemand de la seconde moitié des années 1920. Les universitaires allemands, par exemple, évitèrent l'Université Strasbourg dans la reprise des relations intellectuelles entre les deux pays. ${ }^{19}$

\section{Frontière et rapprochement franco-allemand dans l'après 1945}

\subsection{Les nouvelles frontières et l'évolution des discours sur la frontière}

Après 1945, avec en mémoire les erreurs de l'après Grande Guerre et sous la pression de la guerre froide naissante, il fallut régler la question de la frontière franco-allemande. Au sud - en Alsace - le rétablissement de la frontière sur le Rhin fut considéré comme une évidence (hormis l'annexion temporaire de Kehl jusqu'en 1949), ${ }^{20}$ mais plus au nord, jusqu'à la frontière néerlandaise, la question fit débat. Le gouvernement français, non sans ambiguité, affirma qu'il n'avait pas de velléités annexionnistes. Il entendait initialement séparer la rive gauche du Rhin du reste de l'Allemagne pour assurer sa sécurité, intégrer économiquement la Sarre à la France et internationaliser la Ruhr. Si le gouvernement français maintint officiellement ses exigences jusqu'à la conférence de Moscou en 1947, notamment pour tenir compte d'une opinion française germanophobe, il se rallia courant 1946 au projet d'un futur ensemble ouestallemand. L'idée de construire l'Europe, qui avait déjà fait l'objet de discussions animées dans l'entourage de De Gaulle à Alger en 1943, faisait son chemin. Elle se forgeait autour de l'espace rhénan. Le plan Schuman du 9 mai 1950 fut une étape décisive. Pour illustrer la communauté européenne du charbon et de l'acier, le caricaturiste ouest-allemand Klaus Pielert représenta Robert Schuman cousant solidement, à la machine, la frontière franco-allemande. $^{21}$

Il fallait encore apaiser les inquiétudes de l'opinion publique française. Le résultat du plébiscite sarrois de 1955 , impliquant le retour de la Sarre à l'Allemagne, fut accueilli avec soulagement en Alsace où l'on redoutait que cette affaire ne ravive l'irrédentisme allemand. Quelques mois plus tard, Carlo Schmid, vice-président du Bundestag et figure majeure du SPD, déclara explicitement, le 23 septembre 1956 : «Les hommes de l'Alsace se sont décidés pour la nation française au moins depuis la Révolution [...]. Depuis lors ils

19 Cf. Defrance, Corine : Sentinelle ou Pont sur le Rhin? Le Centre d'Études Germaniques et l'apprentissage de l'Allemagne en France (1921-2001), avec la collaboration de Christiane FalbisanerWeeda, Paris : CNRS Éditions, 2008, p. 67-77.

20 Cf. Stüwe, Hartmut : Evakuierung, Besetzung, Freigabe. Kehler Stadtgeschichte, 1944-1953, Kehl : Kultur-und Verkehrsamt, 2003.

21 Cf. Pielert, Klaus : «Erfinderstolz: Hoffentlich entwickelt sich das Ding besser als die Sicherheitsnadeln », ds. : Rhein-Echo, Düsseldorf, 07/07/1950. 
veulent être et sont effectivement des fils et des citoyens de la nation française. Leur capitale s'appelle Paris. Ce sont des Français ».22

Ces actions de Schuman ou Schmid sont des moments clés du processus d'apprentissage du rapprochement et de la réconciliation des années 1950. L'histoire de la fondation de l'Institut historique allemand de Paris en est l'un des témoins parce qu'elle montre l'évolution du discours sur la frontière. Le discours inaugural de Eugen Ewig, le premier directeur, le 21 novembre 1958, reflète cette attitude : en faisant référence à la devise et au blason de la ville de Paris « Fluctuat, nec mergitur », et à l'adresse du nouveau centre de recherche, rue du Havre, il décrivit la route de la 'barca' venant d'Allemagne et contournant les brisants administratifs pour arriver à bon port à Paris. ${ }^{23}$ En citant des historiens comme Henri Pirenne et Wilhelm Levison, Ewig inscrivit volontairement l'Institut historique allemand dans un courant historiographique dépassant les frontières nationales : il fallait cesser de présenter le Rhin comme un fleuve découpant l'espace et séparant les hommes. Dans la tradition de Lucien Febvre, il fit des voies d'eau des espaces communicants favorisant les échanges socio-culturels et imbrications intellectuelles, et du Rhin un fleuve européen. En dénationalisant l'espace, il participa à sa réorganisation et à sa redéfinition mentale.

\subsection{Vivre à la frontière : vivre avec le voisin ?}

Une note d'information de la préfecture de Strasbourg de 1953 précisait: "Une frontière ne doit pas former, pour ceux qui y habitent, la fin d'un monde ». ${ }^{24}$ Dans les années 1950, le risque de la frontière comme borne des 'cartes mentales' était encore bien réel. Ce qui ressort d'une première étude sur les régions frontières, c'est la grande diversité des situations et la variabilité des résultats en fonction des critères retenus pour les saisir. Ainsi, si l'on considère la conclusion des premiers jumelages franco-allemands jusqu'en 1963 qui sont un des gestes les plus symboliques du rapprochement bilatéral par la base - il s'avère que la majeure partie des cités allemandes concernées était située dans les Länder frontaliers de la Rhénanie-Palatinat et du Bade-Wurtemberg. ${ }^{25}$ En revanche, aucune ville sarroise n'était encore jumelée avec une

22 Cité d'après Defrance, Corine : La dimension régionale dans le rapprochement francoallemand. L'Alsace face à l'Allemagne de l'immédiat après-guerre au début des années 1970, ds. : Dénéchère, Yves/Vincent, Marie-Bénédicte (dir.) : Vivre et construire l'Europe à l'échelle territoriale de 1945 à nos jours, Bruxelles : Peter Lang, 2010, p. 145-158.

23 Cf. Pfeil, Ulrich : Vorgeschichte und Gründung des Deutschen Historischen Instituts Paris. Darstellung und Dokumentation, Ostfildern : Thorbecke, 2007 (Instrumenta, vol. 17), p. 432-434.

24 Archives départementales du Bas-Rhin, 544D220, note d'information, préfecture de Strasbourg, 16/06/1953.

25 Cf. Defrance, Corine : Les jumelages franco-allemands : aspect d'une coopération transnationale, ds. : Vingtième Siècle 99 (2008), p. 189-201. 
commune française. Le poids des tensions suscitées par la question du statut de la Sarre puis la campagne du référendum avaient laissé des traces. Pourtant, si l'on considère la coopération intellectuelle et universitaire, la Sarre avait incontestablement eu un rôle pionnier, que l'on pense ici à la radio sarroise ou à l'Université de la Sarre. ${ }^{26}$ Côté français, la Lorraine et l'Alsace se plaçaient loin derrière la région parisienne ou la Bourgogne pour le nombre de jumelages avec des villes allemandes. Si Nancy et Karlsruhe et Metz et Trèves se jumelèrent respectivement en 1955 et 1957, Strasbourg ne se lia à Stuttgart qu'en 1962. L'hypothèse d'une difficulté alsacienne particulière à entrer en relation avec le voisin allemand est confirmée par un propos de Pierre Pflimlin, alors maire de Strasbourg, déconseillant en 1963 au consul général de la RFA à Nancy l'ouverture d'un Institut Goethe dans sa ville comptant encore «trop de méfiants et de malveillants ». ${ }^{27}$ Le principal problème à l'égard du voisin résultait de ce qu'on appelle le 'malaise alsacien'. La situation devient suraiguë à l'issue du procès de Bordeaux, en 1953, jugeant les coupables du massacre d'Oradour-sur-Glane. Parmi les inculpés se trouvaient douze incorporés de force alsaciens. ${ }^{28}$ Avec leur condamnation, les Alsaciens eurent le sentiment d'être incompris. Avec leur amnistie s'instaura une profonde crise entre Alsaciens et Limousins, voire 'Français de l'Intérieur'. Ce procès laissa des traces profondes et durables. Les Alsaciens, se sentant considérés comme des 'collabos' par leurs compatriotes, eurent des difficultés à tendre la main au voisin allemand.

Finalement, c'est la nécessité du 'vivre ensemble' dans un même espace qui a permis la reprise de l'échange. La dimension culturelle, portée par le dialecte, le bilinguisme et les intérêts économiques l'ont facilitée. Le statut particulier de la Sarre et son intégration économique à la France ont aussi entraîné des échanges frontaliers dès l'immédiat après-guerre. ${ }^{29}$ Les échanges furent facilités en 1954 par la suppression du visa pour les ressortissants allemands puis par la suppression fin 1956 du passeport entre la France et l'Allemagne ce qui ouvrit la voie au tourisme. ${ }^{30}$

26 Cf. Defrance, Corine : Les relations universitaires franco-allemandes avant 1963. Impulsions institutionnelles et initiatives privées, ds. : Lendemains 27 (2002) 107/108, p. 202-219, ici p. 210 ; Müller, Wolfgang : Die Universität des Saarlandes in der politischen Umbruchsituation 1955/56, ds. : Hudemann, Rainer/Jellonek, Burkhard/Rauls, Bernd (dir.) : GrenzFall. Das Saarland zwischen Frankerch und Deutschland 1945-1960, St. Ingbert : Röhrig, 1997, p. 413-425.

27 Politisches Archiv/Auswärtiges Amt : B. 96, vol. 876, note de Hans Günther, consul de la RFA à Nancy, 24/07/1963.

28 Cf. Vonau, Jean-Laurent : Les Malgré-Nous et le drame d'Oradour, Le procès de Bordeaux, Strasbourg : Éditions du Rhin, 2003.

29 Cf. Wille, Christian : Grenzgänger und Räume der Grenze. Raumkonstruktionen in der Großregion SaarLorLux, Frankfurt/M. : Peter Lang, 2012.

30 Defrance : La dimension régionale dans le rapprochement franco-allemand. 


\section{Frontière dépassée - Frontière européanisée ?}

Historiquement, c'est dans l'espace rhénan qu'ont été expérimentées les premières formes d'autodépassement de la frontière, ensuite encouragées et développées par Bruxelles, notamment dans le cadre des programmes Interreg depuis les années 1990. La frontière franco-allemande et franco-germanosuisse a été le berceau des coopérations transfrontalières et des euro-régions. Depuis 2013, c'est aussi en région frontière - en Sarre - qu'est entrepris l'un des projets les plus novateurs et ambitieux. La Frankreichstrategie, en s'appuyant principalement sur la nouvelle génération, vise à faire du Land un territoire bilingue, véritable 'porte de la France' et 'pont vers l'Allemagne', à l'horizon $2043 .{ }^{31}$

\subsection{Apprivoiser la frontière franco-allemande pour construire l'Europe}

Dès 1946, mu par le souci de trouver de nouvelles voies pour assurer la paix, la sécurité et la prospérité en Europe, le ministre néerlandais et politologue Willem Johan Rudolf Thorbecke (1892-1989), dans un ouvrage ressuscitant l'expression de Lamartine "Rhin, Nil de l'Occident », développa l'idée du bassin rhénan comme noyau d'un groupement politique et économique plus vaste, misant sur la « cohésion supranationale » des pays riverains. ${ }^{32}$

L'idée d'une Europe qui abolirait les frontières des États-nations était bien présente dans le milieu fédéraliste. Elle était populaire chez les jeunes et s'exprimait par des actions symboliques spectaculaires. Ainsi, le 6 août 1950, de jeunes Européens firent brûler les barrières de bois à un poste-frontière franco-allemand près de Wissembourg pour réclamer la création d'un parlement, d'un traité et d'un passeport européen. Il y eut ensuite de nombreuses répliques dans d'autres petits postes-frontières. L'un des participants à ces actions d'août 1950 fut un certain Helmut Kohl. Rétrospectivement, ce dernier fit remonter à cet événement fondateur son engagement pour l'intégration européenne. ${ }^{33}$ En 2007, un mémorial européen a été inauguré sur le site de l'ancien poste-frontière. ${ }^{34}$

31 Cf. Staatskanzlei des Saarlandes : Eckpunkte einer Frankreichstrategie für das Saarland, 2014, http://www.saarland.de/dokumente/res_stk/D_Eckpunkte_Frankreich-Strategie_ 210114.pdf (11/01/2017), p. 13. Voir aussi les autres contributions réunies dans ce volume.

32 Thorbecke, Willem Johan Rudolf : La Hollande et le Rhin, ds. : Dumont, Jean (dir.) : Le Rhin, Nil de l'Occident, Paris : Les Editions Sfelt, 1946, p. 297-309.

33 Cf. Kohl, Helmut : Erinnerungen 1930-1982, München : Droemer, 2004, p. 76.

34 Cf. Bock, Hans Manfred [et al.] (dir.) : Les jeunes dans les relations transnationales. L'Office francoallemand pour la Jeunesse, 1963-2008, Paris : PSN, 2008, p. 8. 
En 1951, le Rhin romantique fut le théâtre de la plus importante rencontre européenne de la jeunesse : 35000 jeunes, essentiellement français et allemands, s'y retrouvèrent à l'été. La rencontre de la Loreley avait été conçue comme une première étape dans la voie de la constitution d'un véritable mouvement européen. ${ }^{35} \mathrm{Au}$ début des années 1950, la frontière franco-allemande - et symboliquement le Rhin - furent investis d'une valeur particulière dépassant la dimension franco-allemande au profit de l'Europe. Et les jeunes en furent à la fois les acteurs et les destinataires.

\subsection{La coopération frontalière franco-allemande :} vitrine de la coopération européenne?

Depuis les débuts du processus d'intégration européenne, l'axe rhénan en a constitué la colonne vertébrale géographique et économique. Au niveau local, la frontière relie par la pratique linguistique, par les complémentarités économiques, par les liens affectifs et familiaux. 36

Les échanges se densifièrent au point que plusieurs projets de coopération transfrontalière furent élaborés dès le tournant des années 1960 et 1970 . Ils furent les ancêtres de l'eurorégion du Rhin supérieur et de l'eurodistrict Regio PAMINA. Certaines sont très vastes comme l'euroregio SaarLorLux ou la Grande Région, plus circonscrites comme la Regio PAMINA (Palatinat, Mittlerer Oberrhein, Alsace du Nord), ou bien, locales comme l'eurodistrict Strasbourg-Kehl.

Birte Wassenberg a mis en lumière le caractère pragmatique de ces coopérations initiales. Il s'agissait par exemple, entre villages voisins, de régler des problèmes de voiries ou de traitement des eaux. Le passage de la confrontation à la coopération s'est opéré lentement et, dans le cas de la coopération du Rhin supérieur, la Suisse a joué un rôle d'initiateur et de médiateur dans la fondation de la Regio Basiliensis dès 1963. ${ }^{37}$ Si sa finalité était d'abord économique, la coopération transfrontalière visait à mettre en lumière les facteurs de convergence sur la longue durée aux dépens de ce qui avait divisé les voisins dans l'histoire récente. Dans le cas de l'eurodistrict Kehl-Strasbourg, l'initiative fut lancée dès le début des années 1950 dans le cadre du Conseil de l'Europe ${ }^{38}$ et elle avait aussi pour objectif d'œuvrer au rapprochement. Le projet était alors prématuré et il n'a finalement été réalisé que dans les années

35 Cf. Plum, Jacqueline : Das Europäische Jugendtreffen auf der Loreley im Sommer 1951 : Ein jugendpolitischer Einigungsversuch, ds. : Lendemains 107/108 (2002), p. 190-201.

36 Cf. Piralla, Steve/Barbier, Isabelle : Mariages lorrains. Des frontières qui tissent des liens, ds. : INSEE, Economie. Lorraine $\mathrm{n}^{\circ} 314$ (06/2013).

37 Cf. Wassenberg, Birte : Vers une eurorégion? La coopération transfrontalière franco-germano-suisse dans l'espace du Rhin supérieur de 1975 à 2000, Bruxelles : Peter Lang, 2007.

38 Cf. Wassenberg, Birte : Histoire du Conseil de l'Europe (1949-2009), Bruxelles : Peter Lang, 2012 , p. 75 . 
1990. Tirant le bilan de ces coopérations transfrontalières, Wassenberg conclut : « Konfrontationen wurden dadurch nicht ganz verhindert, aber es wurde eine feste Plattform geboten, auf der diese diskutiert, verhandelt und überbrückt werden können ». ${ }^{39}$

Lucien Febvre estimait que c'était moins le contrôle exercé que les émotions ressenties qui caractérisaient la frontière et que le Rhin, en raison de cette charge émotionnelle, avait été des décennies durant «l'otage des hommes » ${ }^{40}$. Claire Gantet et Bernhard Struck lui donnent raison, mais seulement à partir de 1806/1815. Auparavant la frontière politique ne suscitait guère d'émoi. La frontière était perçue comme espace de transition. C'est au cours du XIX ${ }^{\mathrm{e}}$ siècle que la frontière franco-allemande devint pour les deux voisins le symbole du choc des nations bientôt qualifiées 'd'ennemis héréditaires'. La haine s'y est cristallisée. L'émotion a-t-elle disparu avec la frontière apaisée de l'après 1945 ? Sans doute pas à en juger par la destruction des installations frontalières à l'été 1950. Mais elle a changé de nature. Ce fut alors l'exaltation de brûler une 'barrière' pour ouvrir la voie à l'Europe au nom de la paix et du rapprochement. Cependant, il faut se garder de surinvestir la frontière, et bien des coopérations à la frontière ont été initiées par pragmatisme pour rapprocher les peuples blessés et séparés par l'histoire récente. Aujourd'hui encore, le vivre ensemble peut s'avérer parfois un peu délicat alors que la France et l'Allemagne se trouvent dans des situations économiques et sociales différentes et que la question de la migration et du contrôle des frontières est revenue sur le devant de la scène. Mais force est de constater que la frontière garde une valeur symbolique particulière dans les relations franco-allemandes. Le vocabulaire et la représentation iconographique en témoignent : la coupure, la barrière, la cicatrice sont devenues des coutures et des ponts. D'une part, au niveau 'national', la frontière est l'emblème de son propre dépassement. Comme elle avait autrefois symbolisé l'antagonisme, elle devint le lieu métaphorique de la réconciliation, inséparable de la construction européenne. Elle est souvent identifiée au Rhin, lieu de mémoire français, allemand, francoallemand et européen par excellence. D'autre part, la frontière franco-allemande est devenue un modèle de coopération transfrontalière en Europe et la vie à la frontière a changé : on s'y sent moins à la périphérie de la France et de l'Allemagne qu'au cœur de l'Europe et peut-être même d'un territoire mixte franco-allemand ou rhénan comme l'illustre aujourd'hui la 'Stratégie France' de la Sarre.

39 Wassenberg: Grenzorte, p. 366.

40 Cité d'après Suckow : Deutscher Rhein, französischer Rhein, p. 1. 


\section{Bibliographie sélective}

Beaupré, Nicolas : Le Rhin. Une géographie, Paris : La documentation française, 2005 (Documentation photographique, dossier $\left.n^{\circ} 8044\right)$.

Becker, Nicolaus : Der freie Rhein, d'abrod ds. : Trierische Zeitung 257, 18/09/1840.

Becker, Nicolaus : Gedichte, Köln : DuMont-Schauberg, 1841.

Bock, Hans Manfred [et al.] (dir.) : Les jeunes dans les relations transnationales. L'Office franco-allemand pour la Jeunesse, 1963-2008, Paris : PSN, 2008.

Defrance, Corine : Les relations universitaires franco-allemandes avant 1963. Impulsions institutionnelles et initiatives privées, ds. : Lendemains 27 (2002) 107/108, p. 202-219.

Defrance, Corine : Les jumelages franco-allemands : aspect d'une coopération transnationale, ds. : Vingtième Siècle 99 (2008), p. 189-201.

Defrance, Corine : Sentinelle ou Pont sur le Rhin? Le Centre d'Études Germaniques et l'apprentissage de l'Allemagne en France (1921-2001), avec la collaboration de Christiane Falbisaner-Weeda, Paris : CNRS Éditions, 2008.

Defrance, Corine : La dimension régionale dans le rapprochement franco-allemand. L'Alsace face à l'Allemagne de l'immédiat après-guerre au début des années 1970, ds. : Dénéchère, Yves/Vincent, Marie-Bénédicte (dir.) : Vivre et construire l'Europe à l'échelle territoriale de 1945 à nos jours, Bruxelles : Peter Lang, 2010, p. 145-158.

Freund, Wolfgang : Das wissenschaftliche Institut der Elsass-Lothringer im Reich an der Universität Frankfurt a. M. (1920-1945), ds. : Pfeil, Ulrich (dir.) : Deutsch-fran₹ösische Kultur-und Wissenschaftsbeziebungen im 20. Jahrbundert. Ein institutionengeschichtlicher Ansat\%, München: Oldenbourg, 2007, p. 47-71.

Jeismann, Michael : Das Vaterland der Feinde. Studien zum nationalen Feindbegriff und Selbstverständnis in Deutschland und Frankereich 1791-1918, Stuttgart : Klett-Cotta, 1992.

Kohl, Helmut : Erinnerungen 1930-1982, München : Droemer, 2004.

Lamartine, Alphonse de : La Marseillaise de la Paix, ds. : La Revue des deux Mondes 4/26 (1841), p. 794-799.

Mission Opérationnelle Transfrontalière : Frontière France-Allemagne, http://www.espacestransfrontaliers.org/ressources/territoires/frontieres/frontieres-en-europe/frontierefrance-allemagne/frontiere-france-allemagne-2/ (10/01/2017).

Mozer, Alfred : Entwicklungspolitik zu Hause, ds. : Schöndube, Claus (dir.) : Entwicklungsregionen in der EWG, Bonn : Bundeszentrale für politische Bildung (Schriften der Bundeszentrale für politische Bildung), 1973, p. 14-25.

Müller, Wolfgang : Die Universität des Saarlandes in der politischen Umbruchsituation 1955/56, ds. : Hudemann, Rainer/Jellonek, Burkhard/Rauls, Bernd (dir.) : Gren₹-Fall. Das Saarland zwischen Frankreich und Deutschland 1945-1960, St. Ingbert : Röhrig, 1997, p. 413-425.

Musset, Alfred de : Le Rhin allemand, in : La Revue de Paris, 06/06/1841.

Nordman, Daniel : Frontières de France. De l'espace au territoire. XV Te-XIXe siècles, Paris : Gallimard, 1998.

Oncken, Hermann : Nation und Geschichte, Berlin : Grote, 1935.

Perrin, Jérôme: La collaboration entre Emile Gallé et Victor Prouvé, ds. : Annales de l'Est n spécial (2005), p. 199-210.

Pfeil, Ulrich: Eugen Ewig. „Créer un ordre transnational“. Von einem Mittler zwischen Deutschland und Frankreich, ds. : Pfeil, Ulrich (dir.) : Das Deutsche Historische Institut Paris und seine Gründungsväter, München : Oldenbourg, 2007, p. 294-322. 
Pfeil, Ulrich : Vorgeschichte und Gründung des Deutschen Historischen Instituts Paris. Darstellung und Dokumentation, Ostfildern : Thorbecke, 2007 (Instrumenta, vol. 17).

Pielert, Klaus : «Erfinderstolz: Hoffentlich entwickelt sich das Ding besser als die Sicherheitsnadeln », ds. : Rhein-Echo, Düsseldorf, 07/07/1950.

Piralla, Steve/Barbier, Isabelle : Mariages lorrains. Des frontières qui tissent des liens, ds. : INSEE, Economie. Lorraine ${ }^{\circ} 314$ (06/2013).

Plessen, Marie-Louise von [et. al] : Marianne et Germania. 1789-1889. Un siècle de passions francoallemandes, Paris : Musée du Petit Palais, 1997.

Plum, Jacqueline: Das Europäische Jugendtreffen auf der Loreley im Sommer 1951 : Ein jugendpolitischer Einigungsversuch, ds. : Lendemains 107/108 (2002), p. 190-201.

Politisches Archiv/Auswärtiges Amt : B. 96, vol. 876, note de Hans Günther, consul de la RFA à Nancy, 24/07/1963.

Schöttler, Peter : Geschichtsschreibung als Legitimationswissenschaft 1918-1945, Frankfurt/M. : Suhrkamp, 1997.

Schöttler, Peter : La Zeitgeschichte allemande, ds. : Écrire l'histoire 11 (2013), p. 61-68.

Staatskanzlei des Saarlandes : Eckpunkte einer Frankreichstrategie für das Saarland, 2014, http:// www.saarland.de/dokumente/res_stk/D_Eckpunkte_Frankreich-Strategie_210114.pdf $(11 / 01 / 2017)$.

Struck, Bernhard/Gantet, Claire : Revolution, Krieg und Verflechtung : 1789-1815, Darmstadt : WBG, 2008.

Stüwe, Hartmut : Evakuierung, Besetzung, Freigabe. Kehler Stadtgeschichte, 1944-1953, Kehl : Kulturund Verkehrsamt, 2003.

Suckow, Dirk : Deutscher Rhein, französischer Rhein, ds. : Bundeszentrale für politische Bildung: Geschichte im Fluss, Flüsse als europäische Erinnerungsorte, 11/05/2012, http:// www.bpb.de/geschichte/zeitgeschichte/geschichte-im-fluss/135684/deutscher-rheinfranzoesischer-rhein?p=all3 (10/01/2017).

Thorbecke, Willem Johan Rudolf : La Hollande et le Rhin, ds. : Dumont, Jean (dir.) : Le Rhin, Nil de l'Occident, Paris : Les Editions Sfelt, 1946, p. 297-309.

Vonau, Jean-Laurent: Les Malgré-Nous et le drame d'Oradour, Le procès de Bordeaux, Strasbourg: Éditions du Rhin, 2003.

Walter, François : Les figures paysagères de la nation. Territoire et paysage en Europe (16e-20e siècle), Paris : Editions Ecole des Hautes Etudes en Sciences sociales, 2004.

Wassenberg, Birte : Vers une eurorégion? La coopération transfrontalière franco-germano-suisse dans l'espace du Rhin supérieur de 1975 à 2000, Bruxelles : Peter Lang, 2007.

Wassenberg, Birte : Histoire du Conseil de l'Europe (1949-2009), Bruxelles : Peter Lang, 2012.

Wassenberg, Birte: Grenzorte. Von der Konfrontation zur Kooperation. Das Beispiel des Oberrheins, ds. : Defrance, Corine/Pfeil, Ulrich (dir.) : Verständigung und Versöhnung nach dem „Zivilisationsbruch"? Deutschland in Europa nach 1945, Bruxelles : Peter Lang, 2016, p. 349-368.

Wille, Christian : Grenzgänger und Räume der Grenze. Raumkonstruktionen in der Großregion SaarLorLux, Frankfurt/M. : Peter Lang, 2012.

Zeller, Gaston : Aspects de la politique française sous l'Ancien Régime, Paris : PUF, 1964. 